\title{
XVII. The effect of electrical oscillations on iron in a magnetic field
}

\author{
W.H. Eccles D.Sc. A.R.C.S.
}

To cite this article: W.H. Eccles D.Sc. A.R.C.S. (1906) XVII. The effect of electrical oscillations on iron in a magnetic field, Philosophical Magazine Series 6, 12:68, 109-119, DOI:

10.1080/14786440609463523

To link to this article: http://dx.doi.org/10.1080/14786440609463523

曲 Published online: 16 Apr 2009.

Submit your article to this journal $\sqsubset \pi$

Џll Article views: 2

Q View related articles $\sqsubset$ 


\section{[ 109$]$}

XVII. The Effect of Electrical Oscillations on Iron in a Magnetic Field. By W. H. Eccles, D.Sc., A.R.C.S.*

$T$ THE following investigation of the action of high-frequency oscillations upon iron held magnetized in a magnetic field was carried through in July and August of last year, and was undertaken to supplement in a quantitative manner the rather vague information available. Since Marconi $\dagger$ announced in 1902 that feeble electrical oscillations were capable of altering the flux of induction in a piece of magnetized soft iron, a great deal of work has been done in the endeavour to determine what it is that really happens in this process. A glance through the chronicles of these labours reveals a surprising lack of precise data, and shows, in fact, that most of our knowledge of this subject is merely qualitative. This is due, no doubt, to the complicated possibilities accompanying any experimental method that can be devised. One, for instance, among these difficulties, is the shielding of the greater mass of an iron wire, or other sample, by the eddy carrents that are generated in the metal by the oscilations. This skin-effect was, in a way, overcome by Maurain by using oscillations sufficiently vigorous to penetrate to the very core of the iron or steel wire experimented upon. By this means he has shown that if the $I \mathrm{H}$ curve of a sample be dran $w^{-n}$ in the ordinary manner while these very vigorsus oscillations are in operation, the curve obtained is not looped but is the single path obtained by the coalescence, as it were, of the branches due to increasing and decreasing tields. The single curve thus obtained be called, after Dubem, the "normal curve of magnetization." Duhem had previously shown from thermodynamical reasoning that the effect of a long train of slowly damped uscillations superposed on iron at any stage of the cyclic process would be to bring the representative point on the I H diagram towards a unique locus-a locus, he concluded, exactly the same as that obtained by mechanically agitating the iron throughout the cycle. This theory of Duhem's takes no account, of course, of the shielding of the inner parts of the iron from the action of the oscillations; and thus cannot be demonstrated experimentally except by using, as Maurain $\ddagger$ has done, very powerful oscillations.

In the experiments to be described, an endeavour bas been made to turn the difficulty arising from the skin-effect. Oscillations so feeble have been used that they affected only

* Communicated by the Physical Society: read June 22, 1906.

+ Roy. Soc. Proc. lxx. p. 341, July 1902 .

I Maurain gets slightly different curves by mechanical agitation and by oscillations. 
the outermost layers of the iron wires employed. The cores of the iron wires have therefore not been used.

Other and great difficulties arise in the matter of producing oscillations of determinate and invariable character. Maurain, in the greater part of his work on this subject, appears to have used the oscillations that passed through a helix in series with a Leyden jar kept sparking strongly and continuously by means of an induction-coil. Russell *, in some recent experiments, applied to his iron the oscillations passing through a coil connected directly in series with a small induction-coil. This last method appears to the writer to subject the iron to very violent treatment of a nature not easily described accurately ; for how far the mere surgings of secondary current overwhelm in importance the genuine oscillations, it will be difficult to say. Piola $\uparrow$, again, worked with highly damped oscillations, because, he found, such oscillations produce the highest effects. $\mathrm{He}$ has, indeed, following Rutherford, used this fact in determining the damping factor of a circuit. But in the present investigation these sources of indeterminateness have, as far as might be, been avoided by using a single train of waves instead of a continued torrent of such trains, and by using oscillations as little damped as possible.

It would not be to the point to extend this brief survey of previous work till it included that of Walter, of Ascoli, of Arno, and of Foley ; for all these have paid especial attention to oscillations whose magnetic field was in some degree transverse to the main magnetic field. The present paper deals solely with oscillations whose magnetic field is along the direction of the principal field.

In these experiments fairly soft Swedish charcoal iron, aged, and not freshly annealed, was taken a large number of times round any chosen magnetic eycle (as in ordinary magnetic testing) till what may be called a cyclic state was attained. The field was then given any desired value, and the iron submitted to a single train of oscillations. This was managed by generating the oscillations on a helix surrounding the iron wire. The consequent alteration in pole strength was observed by the deflexion of a magnetometer mirror. These processes were all repeated a number of times for each selected point of the cyclic curve. The figures given in the tables below are thus each the mean of a number of observations.

In detail, the apparatus adopted consisted of two straight

* Proc. Roy, Soc. of Edin. Nov. 20, 1905.

$\dagger$ Elettricista, iv. p. 145, May 15, 1905. 
solenoids each of 3270 turns of No. 20 copper wire wound in 6 layers on a length $59 \mathrm{~cm}$. of split brass tube. They were placed horizontally on one and the same magnetic east-west line, but on either side of a mirror magnetometer. The magnetometer-needle was in the common axis of the two solenoids. The solenoids were connected in series, and so adjusted in position that when a large current was passed through them the magnetometer-needle was undisturbed. A liquid resistance, a Weston milliammeter, suitable switehes and commutators, and a battery of six secondary cells, completed the solenoid circuit. The coil destined to be the seat of the oscillations consisted of 1252 turns of No. $26^{\circ}$ copper wire wound in a single layer on $70 \mathrm{~cm}$. of a glass tube $0.5 \mathrm{~cm}$. in external diameter, and had a resistance of about $3 \mathrm{obms}$. The whole coil was wrapped in paraffined paper and pushed into the brass tube of the east solenoid.

Fig. 1.

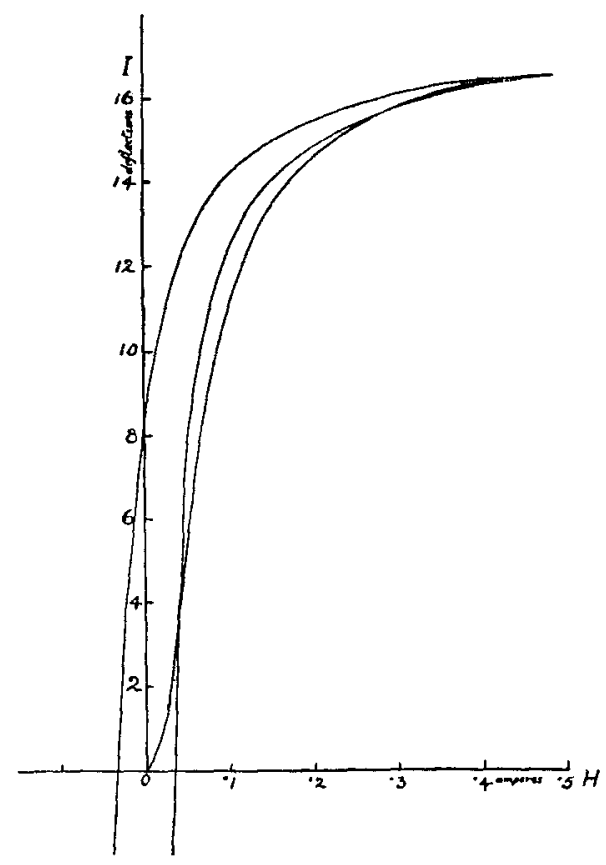

The iron wire examined was unannealled Swedish charcoal iron, diameter $0.749 \mathrm{~mm}$., and was nsed always in lengths of $56 \mathrm{~cm}$. Its characteristic curve is given in fig. 1. The magnetometer was a silk-suspended mirror carrying four very 
small magnets; readings were taken on a scale distant $88 \mathrm{~cm}$.

The object in employing twin solenoids is clear. By placing equal amounts of iron wire in each solenoid and adjusting their positions carefully, the magnetometer deflexion could be kept very slight whatever magnetic variations the iron was taken through, the iron in the one solenoid compensating that in the other.

It was then permissible to exalt greatly the sensibility of the magnetometer. This was done by reducing the controlling field at the needle by means of an auxiliary permanent magnet. As a matter of fact, slight inequalities existing in the construction of the solenoids made perfect balance unattainable; there was always a remanent deflexion, which was different in sign and magnitude for different values of the current through the solenoids, and which in the main proved rather useful by furnishing a check on the proper carrying out of the commutating operations involved in a magnetic cycle.

In the experiments, three iron wires insulated from one another and tied in a bundle were used in each solenoid, the group in the east coil lying, of course, in the glass tube on which the oscillation-coil was wound. The inner ends of the wires were $9 \cdot 3 \mathrm{~cm}$. distant from the magnetometer needle. The field at the needle was reduced to ().034 C.G.S. units. With these arrangements, such a sensibility was attained that on certain days of August last the strokes of distant lightning, not visible in London*, were easily perceptible in the laboratory by their effects on the iron in the oscillation-coil.

The oscillations used were produced by leading the free east end of the oscillation-coil, that is the end distant from the magnetometer, to one side of a micrometer spark-gap. The other end of the oseillation-coil was left insulated. The poles of the spark-gap were connected to the terminals of a diminutive influence machine, that pole not connected to the oscillation-coil being, besides, earthed. Thus, when the handle of the influence machine was turned through a certain angle (depending on the spark-gap) at a speed easily learned, a spark occurred which set up oscillations in the coil. The spark-length finally settled upon was about half a millimetre. It was found possible in this way to get over and over again practically the same magnetometer defexion for every spark, provided the effect of previous oscillations was wiped out by taking the iron through a cycle. As these small sparks were usually inaudible, their occurrence was recognized by the sudden deflexion of the magnetometer-needle.

* The newspapers gave nccounts of thunder-storms in Hertfordshire. 
The calculated period of the stationary waves on the oscillation-coil is $5 \cdot 7 \times 10^{-7}$ second.

A typical set of operations was as follows. The iron was demagnetized by reversals. An amplitude of cycle being then decided upon, the iron was taken a number of times through that cycle. This last operation was stopped at the point settled upon for the observations, and the magnetometer reading taken. A spark was passed, and the magnetometer again read after 30 seconds. A complete cycle was now performed ending at the same point as before, and another spark passed, and so on. Usually, about twenty points on a cycle were examined by 4 or 6 observations at each. In this paper, however, the effects of the spark are recorded as if the observations had been taken only on the ascending half of the hysteresis curve; the figures given being in fact the means of the measured effects at points symmetrical with regard to the origin on the ascending and descending branches.

TABle I.

\begin{tabular}{|c|c|c|c|c|c|c|c|c|c|c|c|c|c|c|c|c|}
\hline $\begin{array}{c}\text { Current in } \\
\text { milliamperes. }\end{array}$ & -150 & -100 & -75 & -50 & -40 & $-30 \mid$ & -20 & 0 & 20 & 30 & 40 & 50 & 75 & 100 & 150 & 200 \\
\hline 50 eycle ... & $\cdots$ & $\ldots$ & $\cdots$ & $\cdots$ & 0.06 & $0 \cdot 10$ & $0 \cdot 13$ & 0.28 & $0 \cdot 38$ & $0 \cdot 45$ & $0 \cdot 60$ & 0.55 & & & & \\
\hline 100 cycle $\ldots$ & $\ldots$ & $\cdots$ & $\cdots$ & $0 \cdot 27$ & ... & ... & ... & $0 \cdot 68$ & 1.08 & $\ldots$ & $\ldots$ & $1 \cdot 26$ & $1 \cdot 30$ & $1 \cdot 18$ & & \\
\hline 150 ercle $\ldots$ & $\ldots$ & $0 \cdot 20$ & $\ldots$ & $0 \cdot 44$ & $\ldots$ & $\ldots$ & $\ldots$ & 0.92 & $\ldots$ & $1 \cdot 32$ & $\ldots$ & 1.51 & $1 \cdot 62$ & 1.57 & $1 \cdot 19$ & \\
\hline 200 cycle... & 0.20 & 0.36 & $\ldots$ & 0.68 & $\ldots$ & $\ldots$ & ... & 1.22 & $\ldots$ & 1.57 & $\ldots$ & $1 \cdot 71$ & $1 \cdot 75$ & 1.55 & 0.93 & $0 \cdot 83$ \\
\hline
\end{tabular}

In this table the figures given can be reduced to absolute measure as follows:- The numbers at the top of each column and down the left column, representing the current through the solenoids in milliamperes, yield when multiplied by 0.0696 the magnetic field in C.G.S. units applied to the iron. The deflexions in the body of the table give the change in polestrength, due to the spark, by multiplying by 0.017 ; or yield the volume-average change of intensity of magnetization by the factor 1.27: or give change of total magnetic moment of the affected specimen by the reducing factor 0.940 .

Fig. 2 is plotted from the above table. The curves show clearly how for increasing cyclic amplitudes, within the range here attempted, the effect of the same spark is increased. The curves show very distinct maxima. It is evident, moreover, that the magnitude of the effect at any point is closely

Phil. Mag. S. 6. Vol. 12. No. 68. Aug. 1906. 
connected with the slope of the hysteresis curve. On the whole the curves tend to corroborate the fact-first noted by

Fig. ¿.

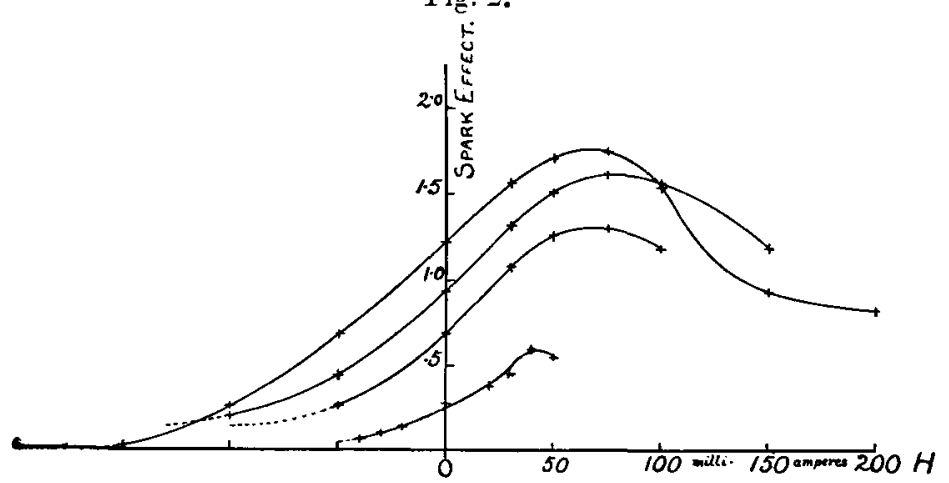

E. Wilson in his 1902 patent specification-that the sensibility of the iron to oscillations is greatest when in the magnetic condition represented by the point of inflexion on the hysteresis curve. To examine this matter more closely

Fig. 3.-The dotted curve shows spark-effect.

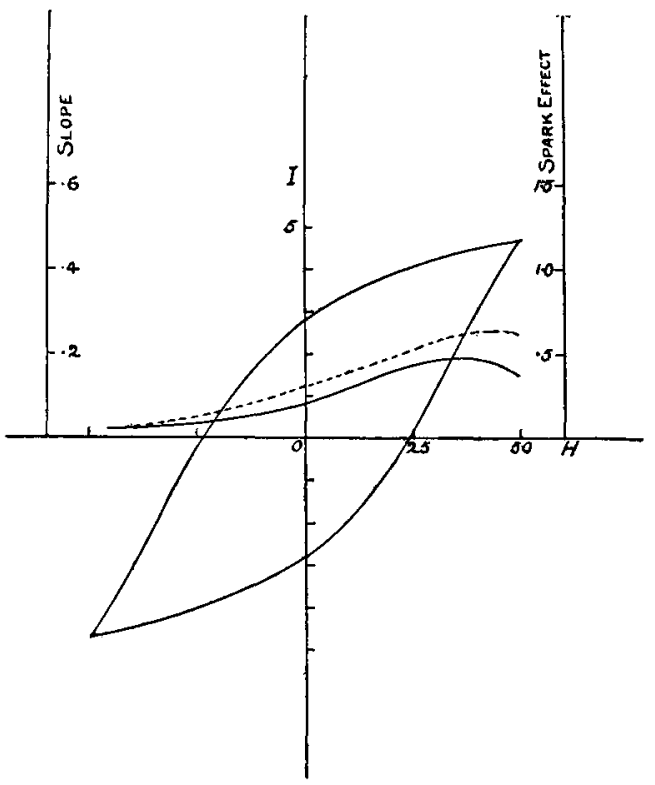

these curves representing the effect of the spark are repeated in figs. $3,4,5,6$, each alongside the gradient curve, that is, 
Oscillations on Iron in a Magnetic Field.

Fig. 4.-The dotted curve shows spark-effect.

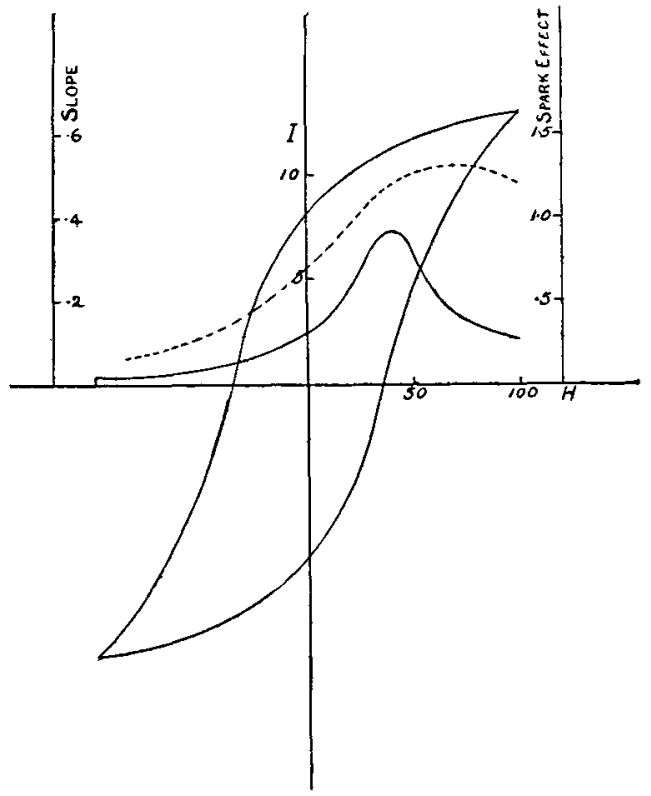

Fig. 5.-The dotted curve shows spark-effect,

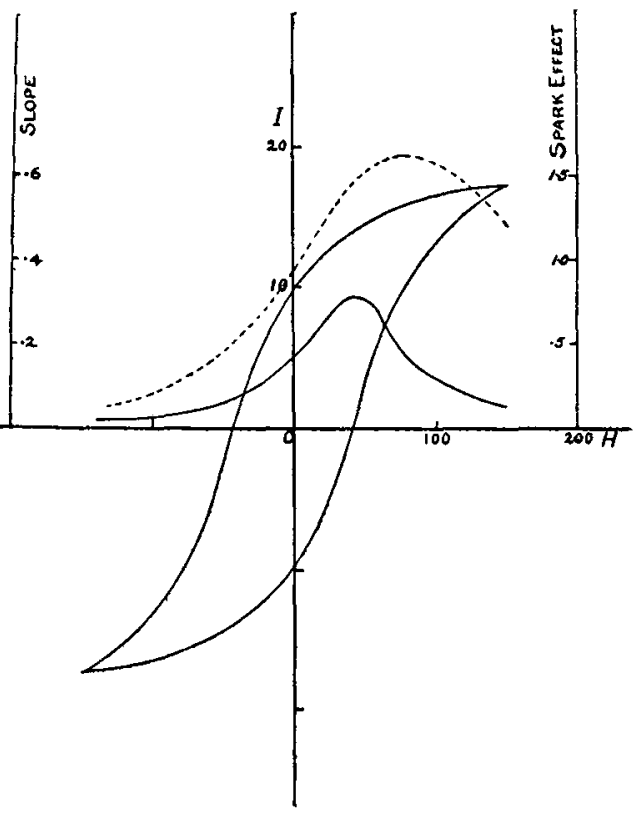


first derived curve, of the corresponding cyclic curve. To assist in picturing the magnetic state of the iron there is plotted on each diagram the proper hysteresis loop. These loops were obtained by independent experiments with the earth's field controlling the magnetometer-needle. (Reducing factor for $I$ is $67 \cdot 5$ ).

Fig. 6.-The dotted curve shows spark-effect.

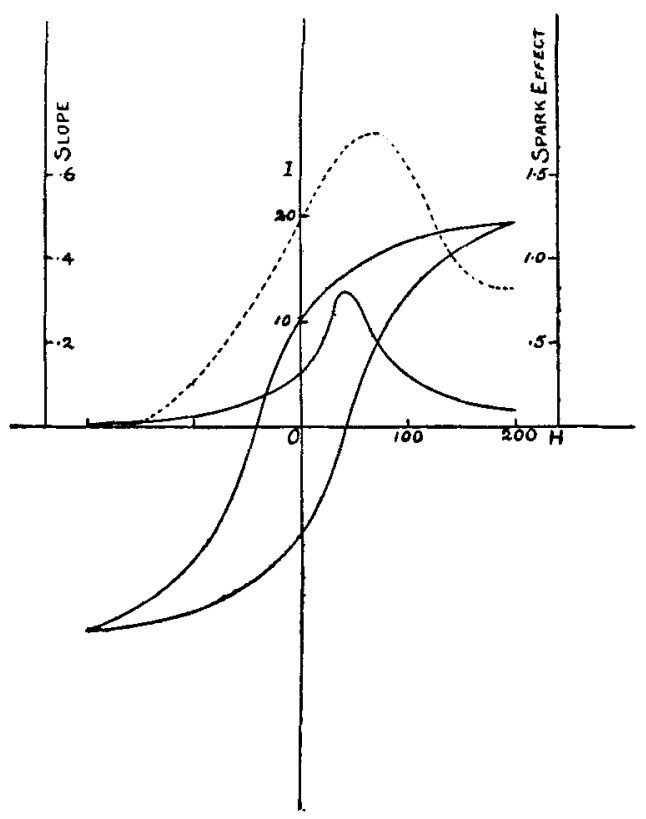

In these figures the abscissæ for all the curves are milliamperes (multiply by 0.0696 to get C.G.S. field). The figures have been so constructed that, except in the cyclic curves, equal ordinates on the different figures represent absolutely equal effects. The reducing factors for the spark-effect curves are as given in connexion with Table I. In all the slope curves the ordinates give at once very approximately the change of intensity of magnetization per $\cdot 001$ C.G.S. increment of field. But the ordinates of the cyclic curve are reduced 21 times in fig. 3, 42 times in fig. 4, 63 times in fig. 5, 84 times in fig. 6 ; and are thus not directly comparable with one another or with the companion curves.

Returning to the spark-effect and the gradient curves, however, it is seen that the spark-effect is by no means a 
simple function of the gradient of the cyclic curve. The curves show, moreover, that the maximum effect of the spark is greater in the cycles of larger amplitude; and not only because the hysteresis curves are steeper in large eycles than in small, but also because in small cycles the effect of the oscillations is, for some unknown reason, much smaller than the diminished gradient would lead one to expect.

It must be mentioned here that during the above measurements the effect of changing the sign of the initial charge given to the oscillation-coil was repeatedly tried. On no occasion was there any perceptible difference in the magnetometer deflexion. The damping of the oscillations must therefore have been very trifling.

A matter quite different, but one of considerable interest, is examined in the following short table of the effect on the magnetometer deflexion of varying the length of the sparkgap from which the oscillation-coil was excited. The observations here recorded were all made at that point of the 0.200 ampere cycle where the magnetic field is zero ; observations made at other points of this or other cycles gave results the same in character.

\section{TABLE II.}

Length of Spark-gap
in centimetres.
$0 \cdot 024$
039
053
070
087

\begin{tabular}{|c|c} 
Magnetometer \\
Deflexions. \\
$0 \cdot 8$ \\
$0 \cdot 8$ \\
$1 \cdot 1$ \\
$2 \cdot 6$ \\
$4 \cdot 4$
\end{tabular}

This table is plotted in fig. 7. On the same diagram appears the curve of Paschen's * observations of potential difference and spark-length.

The most important question to which the results set forth in this paper ean be applied, is that bearing on the real nature of the phenomena observed. A number of observers have summed up their views by the phrase "hysteresis is annulled by oscillations." The present experiments at first sight bear out that conclusion anew. The effect of the oscillations at every point of the cyclic curve is such as to carry the representative point towards the curve that would be yielded by a substance

* Table in J. J. Thomson's 'Recent Researches,' p. 78. 
devoid of hysteresis. This might be interpreted in the same manner as is, usually, the effect of mechanical vibration on iron undergoing varying magnetic forces-the intermolecular bonds that hold the magnetic molecules in their instantaneous configuration are loosened for a moment so that the resultant field can have full play in rearranging the molecular groupings. Looking further into the matter, however, on this view that hysteresis is temporarily annulled, it is evident that on the ascending loop of the cyclic curve the external applied field is permitted to do work when the magnetic shaking occurs. On the other hand, similar alterations of intensity of magnetization occur when the applied field is zero; and, in addition, on the descending loop of the cyclic curve there are alterations in intensity opposite in sense to the applied field. It seems clear, then, that there must exist in the magnetized material intrinsic forces that tend to drag the substance into that presumably more stable magnetic state defined by the "normal curve of magnetization" before mentioned. Thus this view that hysteresis is annulled, taken with the experimental facts of this paper, implies that the store of intrinsic energy of magnetization may possibly be allowed to run down through and during the action of the oscillations. In other words, the action of the oscillations may be analogous to the pulling of a trigger. These considerations threaten to lead us into the often discussed question of the intrinsic energy of a magnetized material having hysteresis. In the present case there is, besides, the difficulty arising from the fact that different layers of the wire used are doubtless differently affected. Notwithstanding, it is perhaps worth while attempting to answer the question: Is the alteration of stored magnetic energy greater than the initial energy of the oscillations producing the alteration?

The latest word on the subject of the intrinsic energy of magnetized iron is contained in a paper by Larmor*. There the expression $2 \pi \int I^{2} d \tau$, where $I$ is the intensity of magnetization and $d \tau$ an element of volume, is deduced for the part of the energy we are here concerned with. Taking the observations recorded in this paper for that point of the -200 ampere cycle where the field is zero, the wastage of the intrinsic energy instigated by a spark is, if it be legitimate to apply here this formula, 3600 ergs. Now the initial energy of the oscillations, which exists as the electrostatic energy of the charge on the oscillation-coil just before the spark-gap breaks down, is about 5700 ergs. These figures are too close to enable a decisive conclusion to be reached; that is, to say

* Roy. Soc. Proc. ]xxi. p. 229, Feb. 1903. 
finally whether or not any considerable amount of magnetic energy is available and drawn upon. Allowing mentally for the oscillation energy lost in Joule effects and in radiation, it seems probable that magnetic work is done by the oscillations-whose efforts, therefore, would appear to be attended with hysteresis loss, just as are those of slower variations of magnetic field.

Table II. and fig. 7 give, in some degree, support to this

Fig 7 .

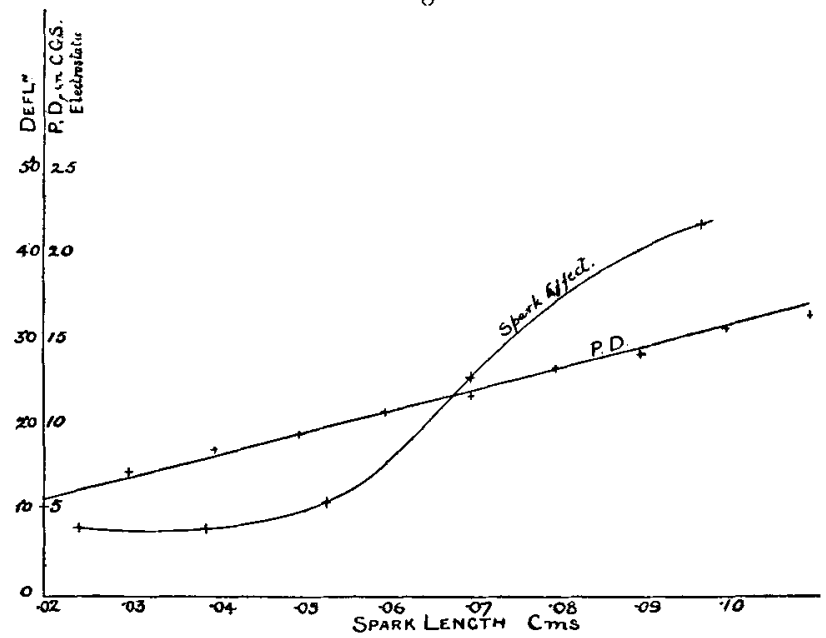

conclusion. The bent (experimental) curve may be taken as representing the magnetic work done by sparks of length shown by the abscissie, other circumstances being the same. The almost straight line represents the potential to which the oscillation-coil was charged at the corresponding spark-length. The relation between the two curves shows that for some portion, at any rate, of the diagram the magnetic work done varies as the square of the charging potential, that is, the electrostatic energy of the initial charge. The bending away of the first curve towards the right is explicable by the consideration, that as the applied oscillations get more violent the outermost layers of the iron wire attain, so to speak, a saturated condition; that is, become incapable of being more greatly impressed--with the result that the magnetic effect of the oscillations begins to fall off in amount. It was for this reason that in the experiments of Table I. the spark-gap was kept at the value 0.53 millimetre, well within the part of the curve free from this saturation effect.

I have to thank my colleague Mr. J. Lister for deducing the gradient curves and drawing all the figures in this paper. 